\title{
Etude Des Determinants Socioeconomiques Et Techniques De La Psciculture Extensive En Cote D'ivoire
}

\author{
Atsé Franck Amian \\ de Recherches Océanologiques (C.R.O), Département Aquaculture, BP V 18 \\ Abidjan, Côte d'Ivoire. \\ National Polytechnique-Felix Houphouet Boigny, Département Eaux, Forêts \\ et Environnement, BP 1093 Yamoussoukro (RCI)
}

\section{Eboua Narcisse Wandan}

Institut National Polytechnique-Felix Houphouet Boigny, Département Eaux,

Forêts et Environnement, BP 1093 Yamoussoukro (RCI)

\section{Melecony Célestin Blé}

Centre de Recherches Océanologiques (C.R.O), Département Aquaculture, BP V 18 Abidjan, Côte d'Ivoire.

\section{Adja Ferdinand Vanga}

Université Peleforo Gon Coulibaly, BP 1328 Korhogo, Côte d'Ivoire.

Pierre Joseph Assi Kaudhjis

Université Alassane Ouattara, BP V 18 Bouaké 01, Côte d'Ivoire.

doi: 10.19044/esj.2017.v13n6p389 ～URL:http://dx.doi.org/10.19044/esj.2017.v13n6p389

\begin{abstract}
The contribution of fish farming is relatively low in the national consumption of fish products. Yet the country has a significant socio-natural potential, which well valued can reduce fish imports and enhance food security. This study, based on a survey conducted in the two main areas of extensive fish farming (Central West and Southwest), was to analyze the technical and socioeconomic determinants of family fish farming to support the dynamic development of this activity. The results showed that $98 \%$ of fish farmers are men of 51 to 60 years old. Most of them are illiterate practicing agriculture as their main activity. Farming infrastructure are usually composed of dams ponds (90\%) of $0.25-1.5 \mathrm{ha}$, and ponds of $0.1 \mathrm{ha}$. Water was provided either by river $(95 \%)$ or groundwater $(5 \%)$. On average most farms are of mall size ( $3 \mathrm{ha}$ ) and were acquired by purchasing lands. Fish farmers mainly practiced mixed farming of Tilapia (Oreochromis niloticus) and Heterotis (Heterotis niloticus). In 60\% of the cases, fish farming is associated with rice cultivation practiced in the shallows. Fish were mainly fed with rice bran. An
\end{abstract}


important number of farmers $(72 \%)$ practiced sexing of in order to improve their yields. Furthermore, $60 \%$ of them have a lower production to 6 tons per year, and fish were old between 1500 and 2000 CFA (1 FCFA=0,0014 euros) per $\mathrm{kg}$ leading to an average annual income of less than 5 million CFA francs.

Keywords: Fish farming, Côte d'Ivoire, pond, dam, Tilapia

\section{Résumé}

La contribution de la pisciculture en Côte d'Ivoire est relativement faible dans la consommation nationale de poisson. Pourtant, le pays possède un potentiel socio-naturel important, qui bien valorisé peut contribuer à réduire les importations de poisson et renforcer la sécurité alimentaire. Cette étude, basée sur une enquête dans les deux principales zones de pisciculture, avait pour objectif d'obtenir des données de base afin d'appuyer la dynamique de développement et de participer à la levée des principales contraintes. Les résultats montrent qu'à $98 \%$, les pisciculteurs sont des hommes de 51 à 60 ans qui sont à $52 \%$ des analphabètes. L'agriculture constitue, dans $62 \%$ de cas leur activité principale. Les infrastructures sont constituées à $90 \%$ barrage-étangs de superficie moyenne $0,25-1,5$ ha et d'étangs de 0,1 ha alimentés à $95 \%$ par de l'eau de rivière ou par la nappe phréatique (5\%). Les exploitations souvent de petite taille ( $3 \mathrm{ha}$ ), ont été acquises dans $53 \%-76 \%$ de cas par achat. La polyculture est la plus pratiquée $(97 \%)$ avec dans $90 \%$ de cas, le couple Tilapia (Oreochromis niloticus)/Hétérotis (Heterotis niloticus). Le son de riz constitue dans $72 \%$ de cas, l'aliment distribué aux poissons. Le sexage dans le but d'améliorer leurs rendements des étangs piscicoles est pratiqué par $72 \%$ des pisciculteurs qui associent dans $60 \%$ de cas, la riziculture à la pisciculture. Par ailleurs, $60 \%$ des pisciculteurs ont une production inférieure à 6 tonnes/an et vendent cette production entre 1500 et $2000 \mathrm{FCFA} / \mathrm{kg}$, ce qui donne un revenu annuel moyen inférieur à 5 millions de francs CFA.

Mots-clés: Pisciculture, Cote d'Ivoire, étang, barrage, Tilapia

\section{Introduction}

En Afrique sub-saharienne le poisson couvre en moyenne 22\% des apports en protéines d'origine animale (Efolé, 2011). Au niveau de Côte d'Ivoire cet aliment représente la principale source de protéines animales consommées, soit près de $15 \mathrm{~kg} / \mathrm{hab} / \mathrm{an}$ (FAO, 2008). La consommation nationale en produits halieutiques est estimée à 278463 tonnes/an, pour une production annuelle d'environ 43532 tonnes, pêches et aquaculture comprises (FAO, 2008). Ces besoins restent en grande partie couverts par l'importation du poisson congelés (MIPARH, 2004 ; FAO, 2008). Ainsi, le pays importe chaque année principalement de la Chine près de 268333 tonnes de produits 
halieutiques pour une valeur de plus de 100 milliards de francs CFA (FAO, 2008 ; MIPARH, 2009).

Ainsi, l'intérêt grandissant porté à l'aquaculture résulte de la baisse des réserves naturelles en ressources halieutiques, occasionnée par la forte pression exercée sur la pêche pour couvrir les besoins de consommation toujours croissants, en rapport avec l'augmentation de la population (Naylor et al., 2000 ; Pauly et al., 2002). L'aquaculture fournit 48\% des apports de poissons pour la consommation humaine et constitue l'une des productions animales ayant la plus forte croissance (FAO, 2012). L'essentiel de cette production provient de l'Asie, particulièrement de la chine qui fournit $70 \%$ de la production mondiale. En Côte d'Ivoire, la contribution de l'aquaculture est relativement faible et ne représente que $1,5 \%$ de la production halieutique nationale (MIPARH 2004, FAO 2008), alors que le pays dispose d'immenses potentialités en termes de ressources en eau pour l'élevage des poissons.

Le pays dispose en effet d'un vaste plan d'eau lagunaire qui s'étend sur une superficie d'environ $1200 \mathrm{~km}^{2}$ qui reste sous exploité. En milieu continental, on note la présence d'environ 1000 petits barrages et retenues d'eau d'une superficie totale de 64000 ha et de milliers de bas-fonds (MINAGRI, 1997).

Spatialement, la production piscicole est inégalement répartie sur le territoire ivoirien, le sud reste la zone où la production est la plus importante avec $52 \%$ de la production totale (527.5 tonnes), suivi du Centre Ouest (160 tonnes) et de l'Ouest (116,5 tonnes), (Bamba, 2002 ; BNETD, 2003). Les petites quantités produites et la stagnation observée depuis 2002 suggèreraient que les systèmes existants, notamment ceux recyclant de la matière organique issue des résidus des cultures et des élevages des exploitations agricoles, sont peu performants et par conséquent non pérennes (Pouomogne et Pemls, 2008). Bien valorisé, ce potentiel socio-naturel peut significativement contribuer à réduire la dépendance du pays vis-à-vis des pêcheries étrangères, à renforcer sa sécurité alimentaire et à alléger la pauvreté en milieu rural (MIPARH, 2004 ; Assi, 2009). Cette étude orientée sur les systèmes piscicoles extensifs vise à comprendre les déterminants socio-économiques et techniques qui les sous-tendent afin de promouvoir durablement le développement de ces systèmes en milieu rural.

\section{Methodologie:}

\section{Localisation et milieu physique des zones d'étude}

L'étude a concerné les deux zones de forte implantation de la pisciculture extensive en Côte d'Ivoire : le Sud-Est et le Centre-Ouest (Fig. 1). La zone de production du Sud-Est couvre une superficie de $28150 \mathrm{~km}^{2}$. Le climat de type subéquatorial est caractérisé par des températures aux amplitudes relativement constantes $\left(29\right.$ à $\left.32^{\circ} \mathrm{C}\right)$, une forte humidité $(85 \%$ en 
moyenne) et des précipitations abondantes de l'ordre de $2000 \mathrm{~mm}$ en moyenne (Avenard et al, 1971 ; Kalms et Kesse, 1977). Cette zone connait deux saisons pluvieuses et deux saisons sèches. La végétation naturelle est caractérisée par la forêt dense qui a laissé progressivement la place à de vastes plantations de cacao et de café. Ainsi, la forêt primaire a pratiquement disparu, en dehors de certaines zones protégées. La densité de la population est de 104 habitants $/ \mathrm{km}^{2}$ (INS, 2014). La population est essentiellement agricole et les principales cultures sont les cultures de rentes : cacao, café, l'hévéa, et le palmier à huile et des cultures vivrières : manioc, mais, taro, igname.

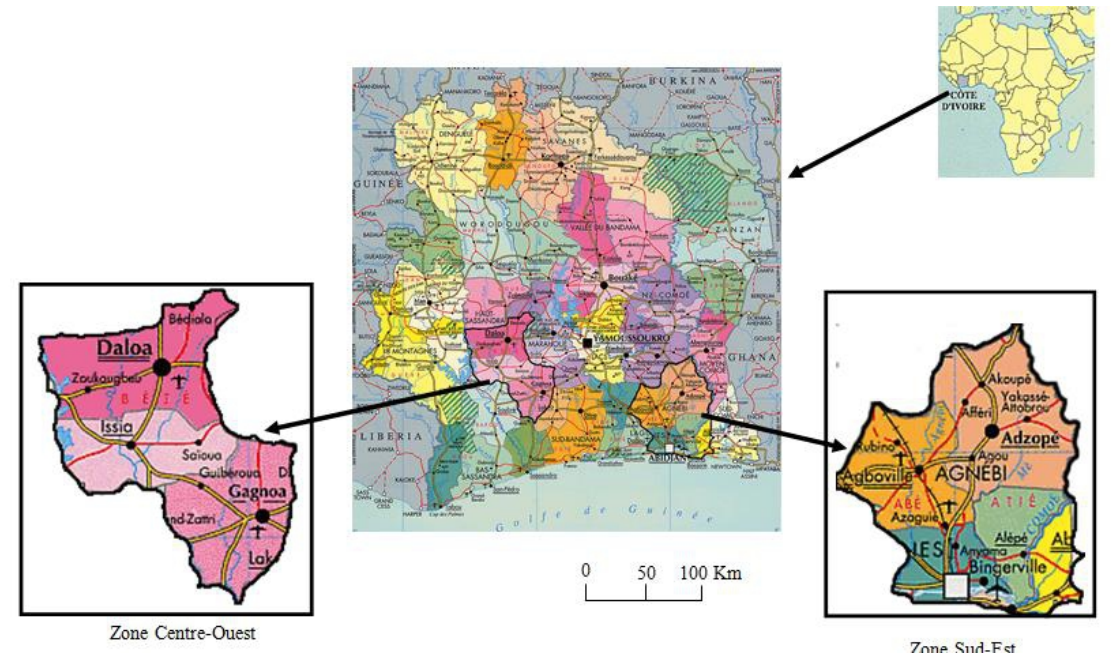

Figure 2 : Détails des zones d'étude

La zone du Centre-Ouest couvre une superficie de $22100 \mathrm{~km}^{2}$. C'est une région forestière en partie montagneuse dont le relief est constitué de hauts plateaux (en moyenne $400 \mathrm{~m}$ ) surplombé par de petites chaines. Le climat de type tropical humide comprend aussi deux saisons de pluies et deux saisons sèches. La moyenne annuelle des précipitations est de $1600 \mathrm{~mm}$ et la gamme de température de 19 à $30^{\circ} \mathrm{C}$ (Avenard et al, 1971). La végétation naturelle est également caractérisée par la forêt dense qui a laissé progressivement la place à de grandes plantations de cacao. La densité de la population de la zone est de 112 habitants $/ \mathrm{km}^{2}$ (INS, 2014) La population est essentiellement agricole avec pour principales cultures celles rencontrées dans le Sud-Est en plus de la riziculture et du maraichage.

\section{La démarche méthodologique}

L'étude s'est focalisée sur des données quantitatives et qualitatives grâce à l'élaboration des questions ouvertes comportant quatre rubriques à savoir : la typologie des producteurs, les systèmes de production, les types d'exploitations, les moyens de production, et la production et les revenus. 
Sur la base des données recueillies sur les acteurs de la filière auprès de l'Association Nationale des aquaculteurs de Côte d'Ivoire (ANAQUACI), 40 exploitations piscicoles ont été choisies sur 302 exploitations présentant une activité piscicole effective dans les zones concernées, soit 13\%. Ce choix a été opéré conjointement avec les responsables de l'ANAQUACI par la méthode d'échantillonnage aléatoire simple

Le logiciel STATISTICA a été utilisé dans le traitement des données quantitatives. Quant aux informations recueillies à partir des entretiens, le traitement a été effectué sur la base du principe de l'analyse du contenu.

\section{Résultats et discussion}

\section{Profils socio-professionnels des exploitants piscicoles}

La figure 2 présente les caractéristiques socio-professionnelles des pisciculteurs enquêtés. On constate que quelle que soit la région, la majorité des pisciculteurs enquêtés (98\%) est de sexe masculin; dans le Centre-ouest aucune femme n'est chef d'exploitation. Ces résultats corroborent avec ceux de Kimou et al. (2016) soit $(97,4 \%)$. Cette dominance des hommes comme chef d'exploitation piscicole pourrait s'expliquer d'une part par le fait que les femmes ne sont pas propriétaires terriens (Tomedi-Eyango et al., 2009). D'autre part, la production piscicole comporte beaucoup de risques et d'incertitude qui font que cette activité est restée réservée aux hommes (Brummett et al., 2010).

La tranche d'âge comprise entre 51 et 60 ans est dominante (43\%) dans les deux régions. Toutefois $36 \%$ ont entre $31-50$ ans dont $37 \%$ dans le SudEst et $33 \%$ dans le Centre-Ouest. Et ces résultats sont en accord avec ceux de Kimou et al. (2016). Ce vieillissement des chefs d'exploitation observé de façon générale pourrait être lié au manque de moyen financier et surtout de subventions qui pourraient stimuler et encourager les plus jeunes à pratiquer l'agriculture au sens large du terme et la pisciculture de manière spécifique. Selon Olowosegun et al. (2004), le fait de constater que 36\% des enquêtés se situent entre 31 et 50 ans, tranche d'âge économiquement active, suggère un meilleur futur pour la production piscicole.

Dans 1'ensemble, les allochtones sont majoritaires (48\%) suivi des allogènes (38\%) dans le Centre-Ouest tandis que dans le Sud-est, les autochtones sont les plus nombreux (63\%). Le taux élevé des allochtones et des allogènes dans le Centre-Ouest pourrait s'expliquer par le fait qu'à la fin des années 50; les nombreux migrants qui ont afflué dans cette zone pour y pratiquer la cacaoculture ont adhéré massivement au Projet Piscicole du Centre Ouest (PPCO) initié entre 1992 et 2000 (Oswald, 1997). Ce projet avait pour objectif de faire de la pisciculture extensive une diversification complémentaire des économies de plantation (Oswald 1997, Assi Kaudhjis, 2005). La prédominance dans le Sud-Est des autochtones pourrait s'expliquer 
par le fait que, ces derniers sont des lagunaires dont la tradition de pêcheurs et le recours au poisson dans l'alimentation des populations se sont perpétuées à travers la pisciculture (Loucou, 1984 ; Perrot, 1989). Selon Kimou et al. (2016), le secteur piscicole ivoirien serait dominé (58.2\%) par les allochtones et les allogènes.

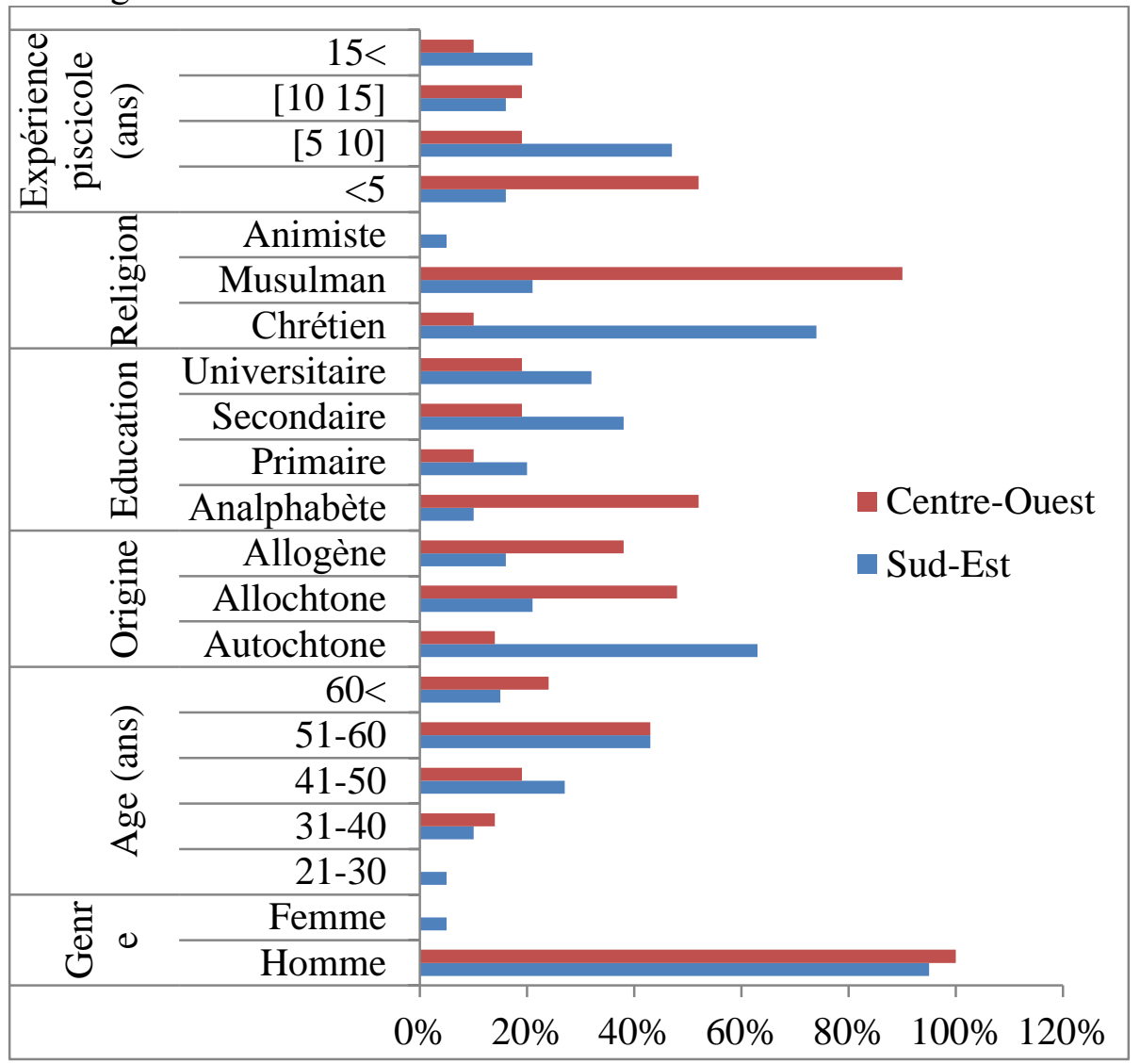

Figure 3 : Quelques caractéristiques socio-professionnelles des pisciculteurs des régions Centre-Ouest et Sud-Est de la Cote d'Ivoire.

S'agissant du niveau d'éducation scolaire, il apparait que 52\% des pisciculteurs sont analphabètes dans le Centre-Ouest alors que dans le SudEst, $20 \%$ sont de niveau primaire et $70 \%$ de niveau secondaire ou plus. Le niveau relativement élevé de la scolarisation observé dans la zone Sud-Est serait lié d'une part à la recherche d'un complément de salaire pour des fonctionnaires et employés d'entreprises au travers du développement de la pisciculture en tant qu'activité secondaire. D'autre part, cela à la reconversion en pisciculteurs de retraités (Efolé, 2011). Le taux d'analphabète élevé (52\%) dans le Centre-Ouest pourrait être justifié par le fait que la majorité des pisciculteurs dans cette zone sont des allochtones ou allogènes pour la plupart 
(86\%). Ces derniers, anciennement ouvriers dans les plantations cacaoyères ont pu acquérir par la suite des terres et bénéficié du projet PPCO.

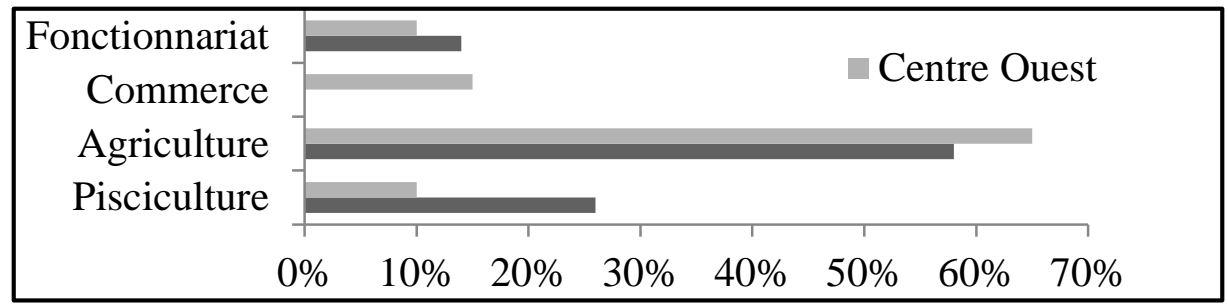

Figure 4 : Activités principales et secondaires des éleveurs dans les régions Sud-Est et Centre-Ouest de la Cote d'Ivoire.

Plus de $22 \%$ des pisciculteurs du Sud-Est ont reçu une formation en pisciculture contre seulement $19 \%$ dans le Centre-Ouest. S'agissant de la participation à des séminaires de formation en pisciculture, 44\% l'ont été dans le Sud-Est contre 19\% dans le Centre-Ouest. L'Islam est la religion dominante parmi les pisciculteurs du centre-Ouest (90\%) tandis qu'au Sud-Est, c'est la religion chrétienne qui domine à $74 \%$.

En termes d'expérience piscicole, la majorité des pisciculteurs enquêtés $(65 \%)$ a une expérience minimum de cinq ans. Ces résultats sont en accord avec ceux de Kimou et al. (2016), soit 66,1\%. Cependant, le Sud-Est enregistre le plus grand nombre de pisciculteurs expérimentés (84\%) contre $48 \%$ dans le Centre-Ouest. Le nombre élevé d'années d'expérience dans la pisciculture peut être un atout dans ce sens que ceux-ci peuvent avoir une bonne appréciation du marché.

En général, l'agriculture constitue l'activité principale de la plupart des pisciculteurs $(62 \%)$ dans les deux régions avec respectivement $58 \%$ pour le Sud-Est et $65 \%$ pour le Centre-Ouest (Fig 3). La pisciculture représente la deuxième activité avec seulement $18 \%$ des exploitants enquêtés. On constate des différences entre régions, c'est ainsi que dans le Sud-Est $26 \%$ en font une activité principale. On constate également que 10 à $14 \%$ des enquêtés sont des fonctionnaires. Ces résultats sont en accord avec ceux de Kimou et al. (2016). Dans le Centre-Ouest, on rencontre quelques commerçants (15\%) qui s'adonnent à cette activité. Cette orientation vers la pisciculture semblerait être liée aux difficultés rencontrées au niveau du binôme café-cacao (persistance des maladies, vieillissement du verger et instabilité du prix de vente). Cette forte diversification des activités découlerait des stratégies adaptatives des enquêtés qui multiplient leurs sources de revenus au travers des activités répondant à la satisfaction des besoins des citoyens, face à la forte poussée démographique des villes (Efolé et al., 2012). 


\section{Les moyens de production}

La figure 4 montre les caractéristiques des étangs utilisés par les pisciculteurs enquêtés. Deux types d'étangs sont généralement utilisés; à savoir : (1) les barrages-étangs qui sont des retenues d'eau ou lacs artificiels ou plan d'eau ou réservoir de collection et (2) les étangs en dérivation. La majorité des enquêtés (90\%) possède au moins un barrage-étang dont $89 \%$ ont une surface moyenne de 0,25 à 1,5 ha. Quel que soit la zone, plus de la moitié d'entre eux (75\%) possède au moins un barrage-étang.

Les barrage-étangs ont des profondeurs variant entre 1,2 et $2,5 \mathrm{~m}$ avec une moyenne de $1,8 \mathrm{~m}$. Pour $64 \%$ des enquêtés de manière générale, la surface de ces barrage-étangs varie à $72 \%$ entre 0,25 et 1 ha dans le Sud-Est et $56 \%$ dans le Centre-Ouest. Le reste des pisciculteurs possède des barrages-étangs dont la surface en eau est plus élevée avec une moyenne de 2,5 ha.

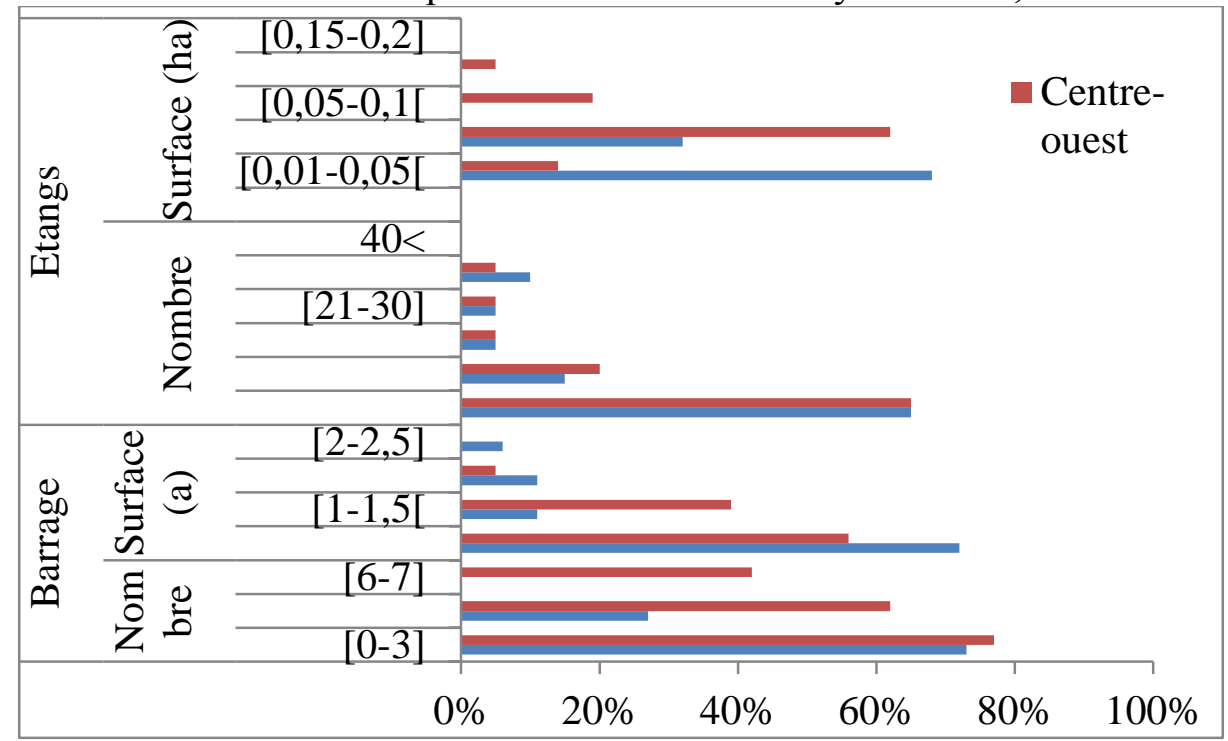

Figure 5 : Caractéristiques des étangs utilisés par les pisciculteurs dans les régions CentreOuest et Sud-Est

Les barrages-étangs du Sud-Est servent à $80 \%$, de réservoir d'eau pour l'alimentation des étangs en dérivation. Dans ces conditions, la pêche y est effectuée au moins chaque trois année. Par contre dans le Centre-Ouest, ceuxci sont majoritairement utilisés pour la production annuelle de poissons.

La figure 4 montre que la majorité des pisciculteurs enquêtés dans les deux régions $(65 \%)$ possèdent 1 à 10 étangs, $18 \%$ en possèdent 11 à 20 dont les superficies varient à $87 \%$ entre 0,01 et 0,1 ha. Un petit nombre de pisciculteurs $(17 \%)$ détiennent 21 à plus de 40 étangs.

Les résultats indiquent que la surface des étangs du Sud-Est varie en moyenne entre 0,01 et 0,1 ha, alors que $24 \%$ des étangs du Centre-Ouest ont des surfaces de plus de 0,1 ha à 0,2 ha. La surface totale en eau de la moitié 
des pisciculteurs enquêtés (50\%) varie entre 1 et 3 ha. Ces résultats sont en accord avec ceux de Kimou et al. (2016). La source d'alimentation en eau de ces structures d'élevage est à $95 \%$ des cas principalement une rivière. Seulement $5 \%$ en moyenne des enquêtés situés tous dans le Centre-Ouest utilise comme source d'alimentation de leurs étangs de l'eau issue de la nappe phréatique.

Les figures 5 et 6 illustrent respectivement la répartition des superficies des exploitations enquêtées par zone ainsi que les différents modes d'acquisition des terres. De manière générale, les exploitations enquêtées sont de petites tailles, dans $63 \%$ de cas, elles sont inférieures à 3 ha. Les exploitations de taille moyenne (4 à $7 \mathrm{ha}$ ) sont majoritaires (36\%) dans le SudEst alors que celles de grande taille (plus de $8 \mathrm{ha}$ ), ont été inventoriées majoritairement dans le Centre-Ouest (24\%).

La faible taille des exploitations piscicoles s'expliquerait par la très grande pression exercée par l'agriculture sur les terres. En effet, dans le SudEst, ce sont les plantations de palmier à huile et d'hévéa alors que dans le Centre-Ouest, ce sont les plantations de cacao et de plus en plus des plantations d'hévéa. La présence d'un petit nombre d'exploitation de grandes tailles (> 8 ha) s'expliquerait par le fait que l'on trouve plus de basfond dans cette zone.

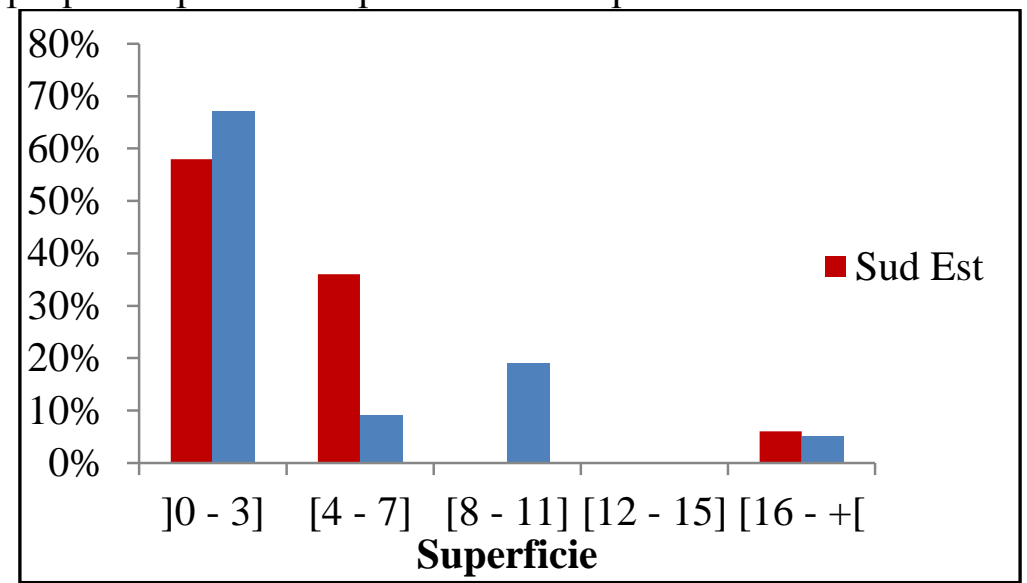

Figure 6: Répartition (\%) de la superficie totale des exploitations par région

Le mode d'acquisition des terres varie suivant les régions, la conception foncière n'étant pas la même dans les deux régions. Dans le Centre-Ouest, il est plus facile d'acquérir les terres de basfonds notamment lorsque celles-ci sont dans la peripherie des grandes villes (Wautelet, 1995). C'est ainsi que l'achat constitue le principal mode d'acquisition des terres dans le Centre-Ouest (76\%) tandis que dans le Sud-Est les terres sont soit un legs $(53 \%)$ ou achetées $(42 \%)$. Le coût d'acquisition peut aller à plus de 250000 $\mathrm{F}$ mais dans $76 \%$ des cas, il est inférieur à $100000 \mathrm{~F} \mathrm{CFA} /$ ha dans le Sud-Est 
et à 63\% entre 100000 et 200 000F CFA dans le Sud-Ouest (le CFA étant égal à 0,0014 euros).

Pour ces terres, les pisciculteurs enquêtés possèdent un titre foncier $(70 \%)$, une attestation villageoise $(37 \%)$ ou un certificat de cadastre $(33 \%)$. On constate que dans le Sud-Est, $47 \%$ possèdent un titre de cadastre alors que la majeure partie (67\%) possède une attestation villageoise dans le CentreOuest. Ces résultats s'expliqueraient par le fait que les pisciculteurs dans le Centre-Ouest étant majoritairement des allochtones, la meilleure façon de pérenniser leurs activités est d'acheter la terre. Comme on le constate, le fait d'acheter la terre entraine une surenchère.

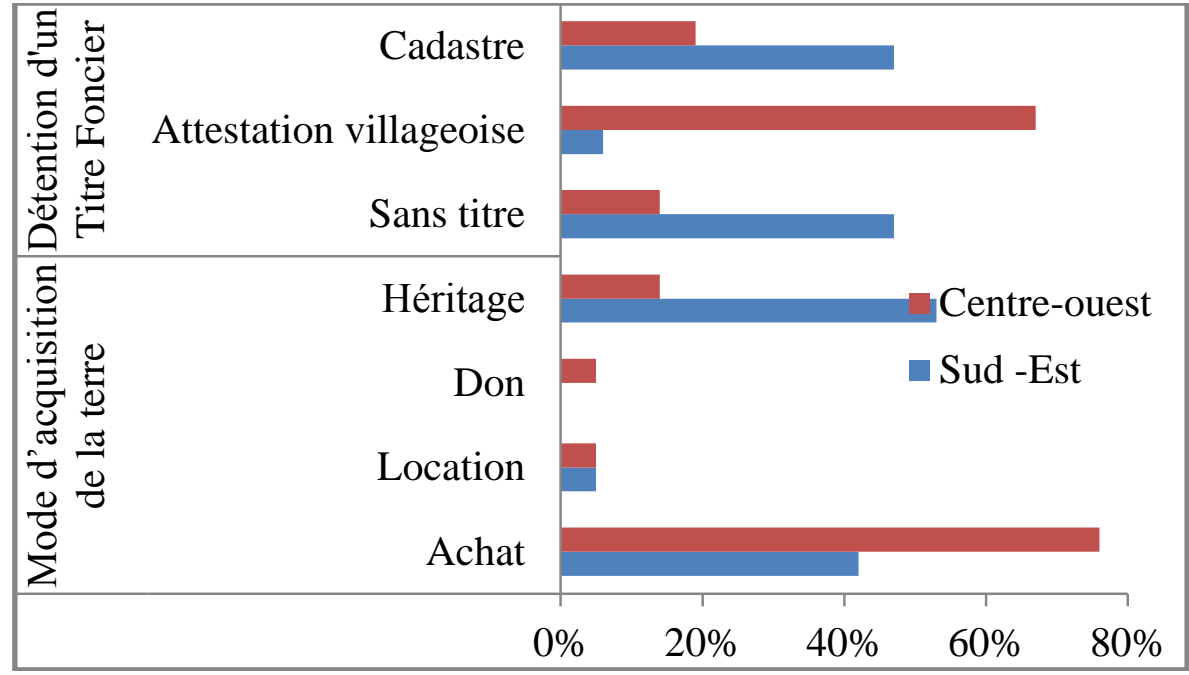

Figure 7 : Mode et type d'acquisition du foncier par les pisciculteurs dans les deux régions enquêtées

Concernant les motivations à la base de l'activité piscicole, elles sont à $82 \%$ le revenu et l'autoconsommation (Figure 7). Ces exploitations ont été majoritairement $(97 \%)$ financées sur fonds propres ; seul un pisciculteur du Centre-ouest a bénéficié d'un don pour la réalisation de son exploitation. On constate que $81 \%$ des pisciculteurs enquêtés dans le Centre-Ouest sont membre d'un groupement d'entraide de pisciculteurs alors qu'aucun enquêté du Sud-Est n'appartient à une association.

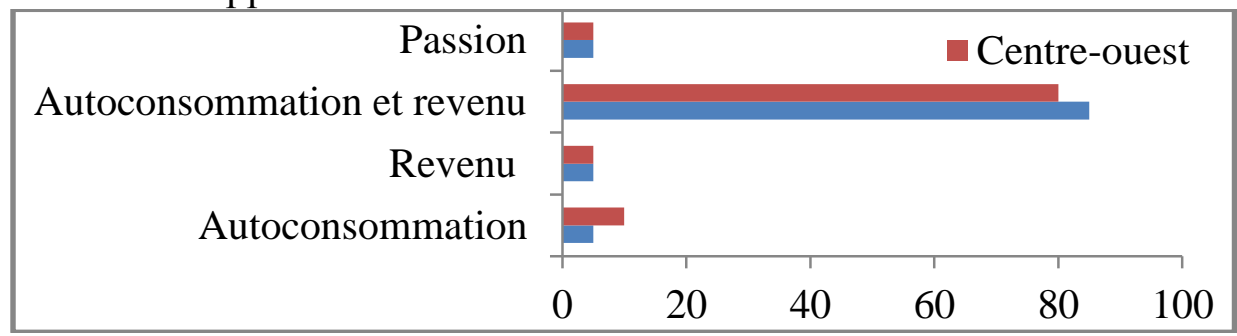

Figure 8 : Motivation des populations pour la pratique de la pisciculture 
La majeure partie des pisciculteurs enquêtés (80\%) n'a reçu aucune formation dans le domaine piscicole. Dans les fermes du Sud-Est, environ 50\% de la main d'œuvre est salariale tandis que dans le Centre-Ouest, elle est familiale (Figure 8). Le niveau de technicité de cette main d'œuvre qu'elle soit familiale ou salariale est estimé moyen par plus de la moitié $(58 \%)$ des chefs d'exploitations. Mais, dans le Sud-Est ce niveau est estimé moyen dans $78 \%$. On constate que $35 \%$ en moyenne des enquêtés reçoivent sur leur site des stagiaires et cela est d'autant plus marqué dans le Sud-Est (48\%) que dans le Centre-Ouest (24\%). En plus, il y a plus de chefs d'exploitation qui ont participé à des séminaires de formation dans le Sud-Est $(44 \%)$ que dans le Centre-Ouest (19\%). Ces formations ont été organisées soit par le Centre de Recherche Océanographique (CRO), la Direction de l'Aquaculture et des Pêches (DAP), le Fonds Interprofessionnel pour la Recherche et le Conseil Agricole (FIRCA) ou l'ANAQUACI durant les dix dernières années.

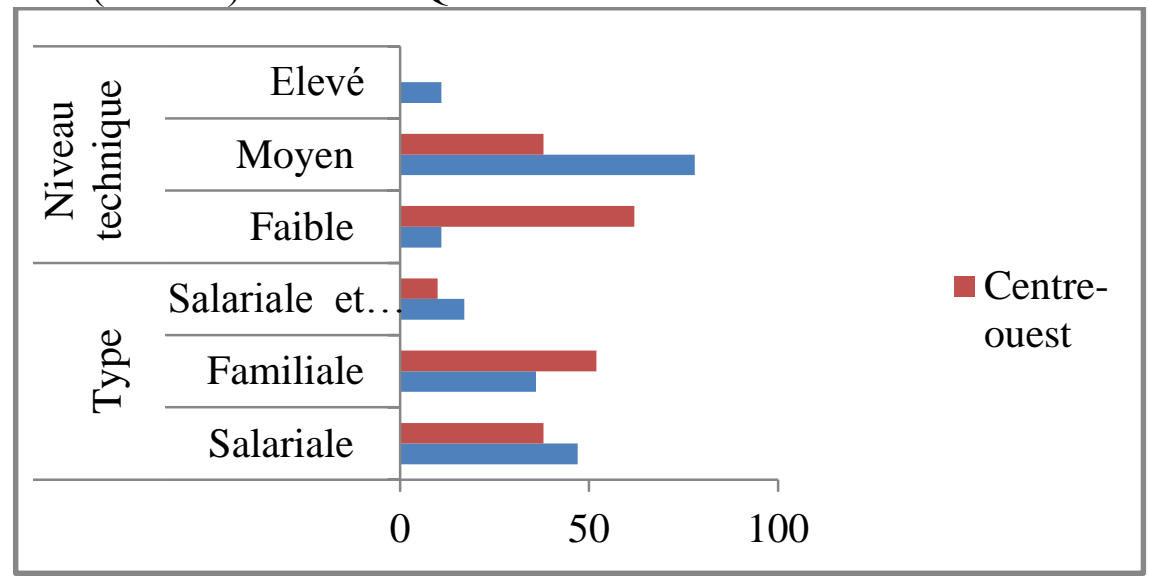

Figure 9 : Type de main-d'œuvre et leur niveau de technicité au niveau des fermes piscicoles

\section{Les systèmes de production}

Comme l'illustre la figure 9, la polyculture, qui consiste à associer des espèces piscicoles aux régimes alimentaires complémentaires afin d'accroître la biomasse produite par un milieu donné, et le cas échéant, pour bénéficier d'effets synergiques entre les différents poissons demeure le type $d^{\prime}$ 'exploitation le plus pratiqué par la majorité des enquêtés $(97 \%)$. Cette pratique apporte une réponse adéquate aux attentes des pisciculteurs car, elle permet d'accroître le revenu final, sans augmenter l'investissement initial ou l'apport d'intrants, si bien que les poissons associés à l'espèce principale sont généralement considérés comme des productions gratuites par les pisciculteurs (Copin et al,, 1993). En outre, la polyculture répond à une stratégie de réduction des risques, puisque les différentes espèces ont des 
exigences d'élevage et des valeurs commerciales différentes (Dabbadie, 1996).

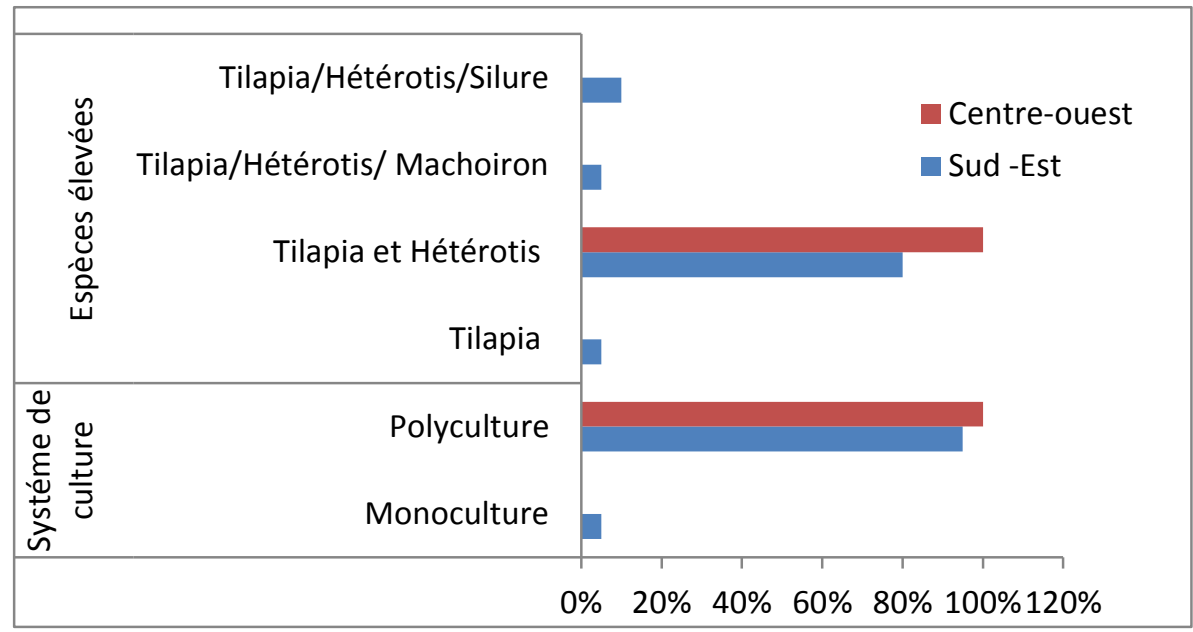

Figure 10 : Associations principales et secondaires d'espèces recensées dans les étangs piscicoles des régions Centre et Ouest de la Cote d'Ivoire

Dans le Centre-Ouest tous les enquêtés pratiquent la polyculture tandis qu'au Sud-Est un seul pratique la monoculture. Le couple Tilapia (Oreochromis niloticus) / Hétérotis (Heterotis niloticus), est essentiellement élevé par la grande majorité des enquêtés $(90 \%)$ dans les deux zones (Figure 9), alors que Kimou et al. (2016) révèlent 58,3\%. Dans le Centre-Ouest tous élèvent ce couple alors que dans le Sud-Est, les pisciculteurs associent à 15\%, au couple Tilapia-Hétérotis, le Machoiron (Chrysichtys nigrodigitatus) ou le Silure (Hetebranchus longifilis). Par ailleurs, un rapport de 1 Tilapia monosexe mâle (Oreochromis niloticus) pour 0,03 Heterotis niloticus, 0,04 Heterobranchus isopterus (ou 1 Clarias sp) et 0,2 Hemichromis fasciatus augmente la production de plus de $40 \%$ par rapport à la monoculture de Tilapia (Dabbadie, 2008).

Le type d'aliment distribué aux poissons par la majorité des pisciculteurs enquêtés (72\%) est essentiellement du son de riz (Figure 10). Environ $20 \%$ des enquêtés utilisent une nourriture à base de trois sous-produits agricoles. Ces résultats sont supérieurs à ceux rapportés par Kimou et al. (2016), qui révèlent $52,2 \%$ pour l'usage de son de riz, $7 \%$ pour la farine basse de riz et $27,8 \%$ pour l'association son de riz et son de maïs dans l'alimentation du poisson en Côte d'ivoire. Dans le Centre-Ouest, $80 \%$ des enquêtés utilisent du son de riz, $10 \%$ associent à cet ingrédient du son de maïs. Le reste des pisciculteurs utilise une composition à base son de riz et de maïs, tourteaux d'arachide, etc. Par contre dans le Sud-Est, 63\% des enquêtés utilisent le son de riz. Le reste utilise une composition à base de farine basse de riz, son de 
riz, farine de poissons, tourteau de soja, tourteau de coton, huile de palme, sel, coquillage $(32 \%)$ ou un aliment industriel $(5 \%)$.

L'utilisation importante du son de riz pourrait être justifiée non seulement par son faible coût mais aussi par sa disponibilité. En effet, on rencontre beaucoup d'unités de décorticage du riz dans les zones de pisciculture. Ces derniers temps, on a observé une hausse du prix du son de riz qui est passé de $100 \mathrm{~F} \mathrm{CFA} \mathrm{/100} \mathrm{kg} \mathrm{en} 1994$ (Sanchez et Glasser., 1996 in Dabbadie, 1996; Dabbadie, 2008) à 1000-2000 F CFA selon qu'il y ait abondance ou pénurie, probablement à cause de la demande.

La durée du cycle d'élevage de ces espèces varie dans les deux régions, pour plus de la moitié des exploitations enquêtées $(65 \%)$, entre 10 et 12 mois mais dans 33-37\% de cas, elle oscille entre 6 et 9 mois (Figure 10). La durée du cycle d'élevage de ceux qui utilisent uniquement le son de riz pour l'alimentation de leurs poissons varie généralement entre 10 et 12 mois alors que dans les élevages où on utilise un aliment composé par le pisciculteur, la durée du cycle d'élevage est plus réduite ; 6 à 9 mois. Ces valeurs corroborent les résultats de Kimou et al. (2016) qui affirment que la faible qualité des sous-produits utilisés justifierait les longues durées de production. En effet, Morissens et al. (1991) considèrent que ces sous-produits agricoles exercent un effet direct d'alimentation de poisson et un effet indirect de fertilisation des étangs. Car selon ces auteurs, le son suscite un faible niveau d'appétence chez le poisson, si bien que l'effet fertilisant est le résultat de la dégradation minérale des fractions non ingérées et non digérées du son. Par ailleurs, associer un fertilisant en petite quantité au son de riz peut induire des rendements élevés (>8 t.ha-1.an- ${ }^{1}$ ) (Morissens et al. 1991), cela pourrait expliquer cette pratique des enquêtés.

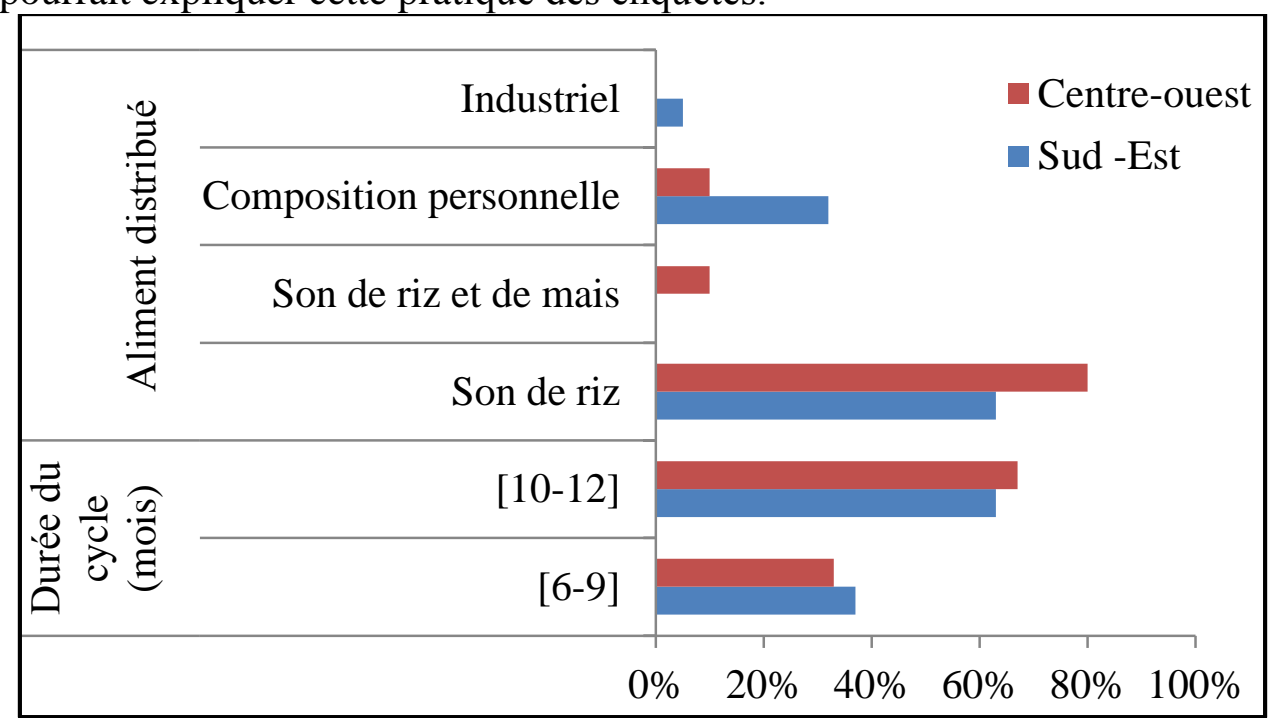

Figure 11 : Durée du cycle des élevages et types d'aliment distribués aux poissons dans les deux régions de la Cote d'Ivoire 
Les résultats indiquent que la majorité des enquêtés (72\%) pratiquent le sexage qui consiste en l'élimination des femelles des étangs afin de favoriser le grossissement des mâles. Cette pratique est utilisée dans le but d'améliorer le rendement des étangs piscicoles.

En termes d'intégration, on observe une prédominance du système production végétale/pisciculture avec comme culture principale la riziculture. En effet, $38 \%$ des pisciculteurs enquêtés dans le Centre-Ouest pratiquent la rizipisciculture et $11 \%$ dans le Sud-Est. L'empoissonnement et le grossissement de poisson en rizière est fondamentalement un système aquacole extensif reposant principalement sur la nourriture présente à l'état sauvage dans les rizières (Halwart et al., 2010). La quantité de poissons pouvant être récoltée dans les exploitations rizipiscicoles varie considérablement en fonction de la densité d'empoissonnement, de la taille des poissons au moment de l'empoissonnement et de l'emploi ou non d'aliments complémentaires. Selon Zhang (1995) et Hem et al. (2001), sans alimentation, la production par récolte peut varier de 100 à $750 \mathrm{~kg} \cdot \mathrm{ha}^{-1} \cdot \mathrm{an}^{-1}$, tandis qu'avec une alimentation, le résultat pourrait se situer entre 1055 et 1 $812 \mathrm{~kg} \cdot \mathrm{ha}^{-1} \cdot \mathrm{an}^{-1}$. L'ensemble des rizipisciculteurs enquêtés nourrissent leur poisson de manière irrégulière avec du son de riz et leur rendement sont en accord avec ceux de Hem et al., (2001). Cultiver simultanément du riz et des poissons optimise l'utilisation du milieu. Les dépenses en sarclage sont réduites et peuvent être éliminées par utilisation de poissons herbivores. La réciprocité entre le poisson et le riz implique des effets mutuellement bénéfiques. Le riz agit comme un bain d'azote et contribue à réduire l'ammoniac qui pourrait être libéré par le poisson, contribuant ainsi à rendre l'eau plus propre pour ce dernier. Le poisson a un effet favorable sur la production du riz dont la quantité peut être augmentée de 10 à 15\%. Cela résulte principalement d'une fertilisation supplémentaire provenant des déjections des poissons, de l'effet d'une alimentation artificielle, d'un meilleur tallage des plants, d'une réduction des insectes nuisibles et d'une meilleure aération du sol (Hem et al., 2001 ; Hilbrands et al., 2004). En outre, cette technique apporte aux cultivateurs et aux populations locales des protéines animales peu coûteuses et un revenu complémentaire non négligeable. L'aspect environnemental transparaît essentiellement à travers le contrôle biologique des plantes adventices, des mollusques et des larves de moustiques dans le champ de riz irrigué. L'élimination des larves d'anophèles et de mollusques par des poissons peut être considérée comme une bonne méthode de lutte contre le paludisme et la bilharziose (Lacroix, 2004).

La fertilisation des étangs par la fiente de poulet, la bouse de bœuf, etc., qui était autrefois pratiquée a été abandonnée par une infirme partie des pisciculteurs enquêtés. On a constaté qu'un seul pisciculteur du Sud-Est a 
introduit l'association porc-pisciculture sur un de ses étangs de grossissement. Le système consiste en un enclos en dur construit en pente légère sur la rive de l'étang. Un canal de drainage permet le lavage du lisier de porc avec de l'eau en provenance de l'étang. L'enclos à sol dur permet de perdre moins de lisier. L'élevage de 1 à 4 porcs par $100 \mathrm{~m}^{2}$ d'étang peut permettre d'obtenir un rendement de 20 à $50 \mathrm{~kg}$ dans une période de six mois (Hilbrands et al., 2004).

$\mathrm{Au}$ vu de ces résultats, Il semble être nécessaire de promouvoir une intensification écologique des systèmes intégrés production végétale-animalepisciculture pour une meilleure durabilité de la pisciculture (Milstein, 2005). Ceci permet de résorber les déchets et effluents issus des productions végétales et animales tout en préservant l'environnement.

L'usage de fiche technique d'élevage qui permet de suivre de manière adéquate la distribution des rations alimentaires, l'évolution du poids des poissons et d'évaluer les paramètres zootechniques de l'élevage n'est pratiqué que par $43 \%$ des pisciculteurs enquêtés.

\section{Production et revenus}

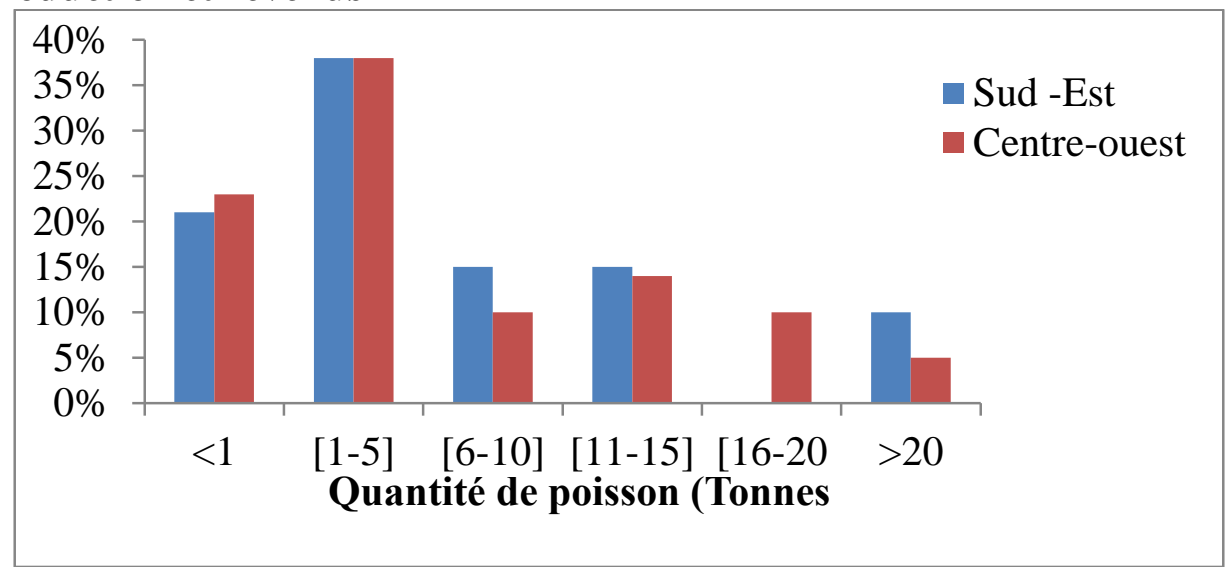

Figure 12: Production des étangs piscicoles dans les deux régions de la Cote d'Ivoire

La figure 11 présente les quantités de poisson produites. De manière générale, le niveau de production piscicole des fermes, reste généralement faible ; dans $60 \%$ de cas, elle est inférieure à 6 tonnes/an dans les deux régions. Dans le Centre-Ouest 34\% des enquêtés produisent entre 6 et 20 tonnes/an tandis que dans le Sud-Est 30\% des enquêtés ont une production qui varie entre 6 et 15 tonnes/an. Par ailleurs, plus de la moitié des exploitations (52\%) a un rendement inférieur à $2 \mathrm{t} / \mathrm{ha} / \mathrm{an}$, Environ $40 \%$ ont un rendement supérieur à $5 \mathrm{t} /$ ha/an dans le Sud-Est. La faible production s'expliquerait en partie par le fait qu'à part un nombre réduit de ferme, la pisciculture demeure une activité marginale dans les deux zones (Pouomogne et al.,2010) et d'autre part par la faiblesse de l'encadrement et des organisations sociotechniques. Selon Kimou et al. (2016) et Burel et Médale (2014), les faibles teneurs en proteines et la 
faible digestibilité des fibres des sous-produits agricoles par les poissons ont pu entrainer ces faibles rendements. Sur cette question, Ouattara (2004) observe une augmentation du gain de masse de $29,49 \%$ et du taux de survie de 60,53\% chez les juveniles de Sarotherodon Melanotheron nourris avec un aliment composé à $30 \%$ de proteines par rapport à une alimentation aux son de riz, de blé et de maïs.

Le prix du poisson est fonction de la région; il est dans $85 \%$, à moins de $1500 \mathrm{~F} \mathrm{CFA} / \mathrm{kg}$ dans le Centre-Ouest et à $89 \%$ de cas entre 1500 et $2000 \mathrm{~F}$ CFA/kg dans le Sud-Est. Pour la majorité (68\%) des exploitations enquêtées, le poisson est vendu essentiellement localement, seulement $25 \%$ d'entre eux vendent leur production en dehors de leur localité (Figure 12).

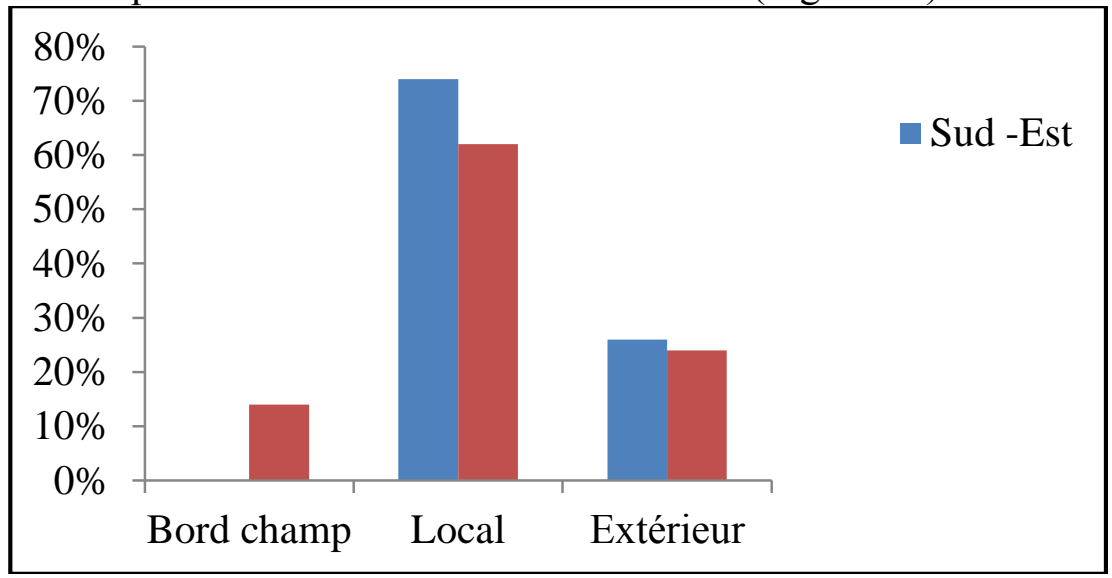

Figure 13 : Lieux de vente du poisson issu des fermes piscicoles du Sud-Est et du CentreOuest de la Cote d'Ivoire

Concernant le revenu annuel des exploitations, pour plus de la moitié des enquêtées (54\%), il est inférieur à 5 millions de francs CFA (Figure 13). Ces valeurs sont accord avec celles de Kimou et al. (2016), qui affirment que ces valeurs moyennes enregistrées de production annuelle (412 666 à 4329 255 F CFA) semblent satisfaire les pisciculteurs qui pratiquent cette activité depuis de nombreuses années. Cependant certains (28\%) ont un revenu variant entre 6 et 15 millions par an, et d'autres (18\%) ont même un revenu supérieur à 16 millions. Dans le Sud-Est le revenu se situe entre 1 et 10 millions de francs CFA/an pour $58 \%$ des enquêtés tandis que dans le Centre-Ouest $48 \%$ ont entre 1 et 10 millions de francs CFA par an. Selon Hecht (2007), la capacité de production de ces pisciculteurs pourrait être améliorée en mettant à leur disposition des aliments de qualité à moindre coût, formulés à partir des matières premières locales accessibles. 


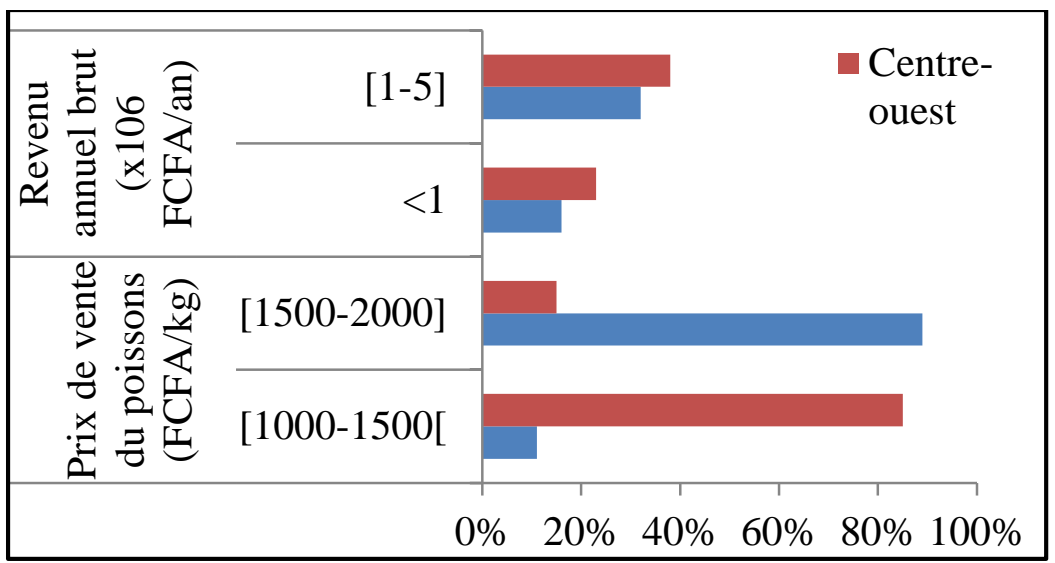

Figure 14 ; Prix de vente du poisson et revenus des pisciculteurs dans les deux régions de la Cote d'Ivoire

\section{Conclusion}

Cette étude a démontré que le contexte socio-environnemental était un facteur important dans la structuration et le fonctionnement des fermes piscicoles dans les deux régions d'étude.

Les exploitants sont vieillissants (51-60 ans en moyenne), l'activité piscicole étant une activité peu attractive et demandant plus d'investissement que l'agriculture.

L'association de l'agriculture est plus pratiquée dans le Centre-Ouest et l'association avec l'élevage, reste encore marginale et artisanale. La productivité est encore très faible en raison de la non maîtrise des techniques de production et de gestion des exploitations. Bien que les rendements soient faibles, le revenu issu de la pisciculture est acceptable et pourrait être lié à la demande sans cesse croissante en poisson. Ce travail n'a pas abordé l'analyse économique de l'activité à cause de la non disponibilité des chiffres fiables, elle-même due au fait que l'activité a été fortement impactée par la crise socioéconomique de ces dernières années. Cet aspect sera abordé dans l'analyse de la chaine de valeur.

Les résultats indiquent que l'utilisation du son de riz demeure l'alimentation de base de la pisciculture dans les deux zones. Les rendements étant faibles, il est urgent de mettre en œuvre une combinaison optimale des différents facteurs de production : valorisation du son de riz, fertilisation plus poussée ; ce qui devrait permettre de réduire les faiblesses.

\section{References:}

1. Assi Kaudjhis J. P., 2009. Evaluation des réglementations et des programmes aquacoles : Côte d'Ivoire. Sustainable Aquaculture 
Research Networks in Sub Saharan Africa (SARNISSA), EC FP7 Project, 52p.

2. Assi Kauhjis (J.P.) 2005 : « étude géographique de l'aquaculture en Afrique subsaharienne: cas de la Côte d'Ivoire ». thèse de doctorat de Docteur en Sciences, orientation Géographie Humaine, de la Faculté des sciences de l'Université Libre de Bruxelles, 353 p.

3. Avenard J. M., Eldin M., Girard G., Sircoulon J., Touchebeuf P., Guillaumet J. L., djanohoun E. \& Perraud A. 1971. Le milieu naturel de la Côte d'ivoire. ORSTOM (Publ.), Paris, france, 391p.

4. Bamba V., 2002 : «Marché et commercialisation du poisson de pisciculture en Côte d'Ivoire » Contrat d'auteur-Rome : FAO. $55 \mathrm{p}$

5. BNETD, 2003. Bilan-Diagnostic et Prospectives pour la relance du secteur pêche et aquaculture en Côte d'Ivoire : Rapport provisoire Abidjan, $123 \mathrm{p}$

6. Brummett R.E, Youaleu J.L.N, Tiani A.M. and Kenmegne M.M., 2010. Women's traditional fishery and alternative aquatic resource livelihood strategies in the Southern Cameroonian Rainforest. Fisheries Management and Ecology, 17, 221-230

7. Burel C. \& Médale F. 2014. Guide de l'utilisation des protéines d'origine végétale en aquaculture. Oilseeds Fats Crops Lipids 21: 115.

8. Copin Y., \& Oswald M., 1993. Orientation des techniques d'élevage de la pisciculture artisanale dans le centre- Ouest de la Côte d'Ivoire. In Banarbé G., Kestemont P. (Eds), Production, environnement and quality. Bordeaux aquaculture 92. EAS spec. Publ., Ghent (Belgium), 18, 407-419

9. Dabbadie L., 2008. L'optimisation de la densité d'empoissonnement d'un étang de pisciculture, Pratique de l'aquaculture/ encyclopédiel www. Aquatrop.fr (consulté le 15/03/2009)

10. Dabbadie L., 1996. Etude de la viabilité d'une pisciculture rurale à faible niveau d'intrant dans le Centre -Ouest de la Côte d'Ivoire : Approche du réseau trophique. Thèse de Doctorat en Océanographie de l'Université Paris 6, Paris (France), 244 p.

11. Efole E. T., Aubin J., Mikolasek O., Corson M. S., Tomedi E. M., Tchoumboue J., van der Werf H.M.G. \& Ombredane D., 2012. Environmental impacts of farms integrating aquaculture and agriculture in Cameroon. J. Cleaner Prod. 28, 208-214. 
12. Efole Ewoukem T., 2011. Optimisation biotechnique de la pisciculture en étang dans le cadre du développement durable des Exploitations Familiales Agricoles au Cameroun. Thèse de Doctorat de docteur de l'institut supérieur des sciences agronomiques, agroalimentaires, horticoles et du paysage de l'Université Européenne de Bretagne, $215 \mathrm{p}$.

13. FAO, 2012. La situation mondiale des pêches et de l'aquaculture ; Département de Pêches et Aquaculture, FAO (Ed), Rome (Italie), 261 p.

14. FAO, 2008. Profil de la pêche par pays, la république de Côte d'Ivoire. FAO, Rome, Italie, 43 p. http://www.fao.org/fishery/facp/CIV/fr (ftp://ftp.fao.org/FI/DOCUMENT/fcp/fr/FI_CP_CI.pdf).

15. Halwart M. \& Modadugu V. G. (Eds.), 2010. L'élevage de poisson en rizière. FAO and WorldFish Center, 98 p.

16. Hecht T. 2007. Reviewof feeds and fertilizers for sustainable aquaculture development in Sub-Saharan Africa. In: Hasan MR, Hecht T, De Silva SS, Tacon AGJ, eds. Study and analysis of feeds and fertilizers for sustainable aquaculture development. Rome (Italie): FAO.

17. Hem S., Curtis M. Y., Sene S., Sow M. \& Sagbla C., 2001. Pisciculture Extensive en Guinée Forestière : Modèle de développement intégré et rizipisciculture. Rapport Final PROJET 7 . Aep. GUI. 104 Convention CEE /IRD, 85p.

18. Hilbrands A. \& Yzerman C., 2004. La pisciculture à la ferme. 3 ed. Wageningen (NL), Fondation Agromisa, 73 p. Agrodok 21.

19. INS., 2014. Recensement Générale de la Population et de l'Habitat. Rapport d'exécution et présentation des principaux résultats. 49p.

20. Kalms J.M. \& Kesse A., 1977. Opération connaissance du climat. Rapport Analytique 1977. Ministère ivoirien de la recherche scientifique, Institut des Savanes, Bouaké, Côte d'Ivoire : 42 p.

21. Kimou N. B., Koumi R. A., Koffi M. K., Atsé C. B., Ouattara I. N., \& Kouamé P. L., 2016. Utilisation des sous-produits agroalimentaires dans l'alimentation des poissons d'élevage en Côte d'ivoire. Cah. Agric. 25, 25006. DOI: 10.1051/cagri/2016012

22. Lacroix E., 2004. Pisciculture en Zone Tropicale. Hamburg, GFA Terra Systems et GTZ. 225p. 
23. Loucou J.N., 1984. Histoire de la Côte d'Ivoire, 1- La formation des peuples, CEDA, Abidjan, 1984, 208 p.

24. Milstein A., 2005. Polyculture in aquaculture Review Article. Animal Breeding Abstracts, CABI (Ed), Wallingford, Oxfordshire (UK), $73(12), 15 \mathrm{~N}-41 \mathrm{~N}$

25. MINAGRI, 1997. Programme d'Appui Institutionnel au Secteur Aquaculture et Pêches, document de travail. Abidjan : DPH, 75 p.

26. MIPARH, 2009. Plan directeur de la pêche et de l'aquaculture (PDPA). Rapport, 84 p.

27. MIPARH, 2004. Annuaire des statistiques de l'Aquaculture et des pêches. Abidjan, 162p.

28. Morissens P., Da Costa S.K., Dembele I., Koffi C., Petel C. \& Lazard J., 1991. Adaptabilité de différents aliments et fertilisants aux conditions particulières des élevages de Oreochromis niloticus en étang dans le milieu rural ivoirien. In : Kaushik S.J. et Luquet P. (eds), Fish nutrition in practice, Biarritz 24-27 juin 1991, INRA (Publ.), Paris, France (972 p) : 717-729.

29. Naylor R. L., Goldburg R. J., Primavera J. H., Nils Kaustky M., Beveridge C. M., Clay J., Folkes C. and Lubchenco J., 2000. Effect of aquaculture on world fish supplies. Nature, 405, 1017-1024

30. Olowosegun, T., Sanni, A. O., Sule, A. M. and Bwala, R. L. 2004. Contribution of women to Fisheries development in Kainji Lake Basin, in 2004 FISON Conference proceedings, Pp 91-97.

31. Oswald (M.), 1997 - Recomposition d'une société au travers de plusieurs crises : La société rurale Bété (Côte d'Ivoire), Thèse de Doctorat, INA Paris-Grignon, 290p + annexes.

32. Ouattara N. I. 2004. Étude du potentiel aquacole d'une population du tilapia estuarien Sarotherodon melanotheron Rüppell 1852 isolée dans le lac de barrage d'Ayamé (Côte d'Ivoire). Thèse de Doctorat, démographie des poissons et hydroécologie, Université de Liège (Belgique), 288p.

33. Pauly D., Christensen V., Guénette S., PitcherT.J., Sumaila R.U., Walters C.J. and Watson R., Zeller D., 2002. Towards sustainability in world fisheries. Nature, 418, 689-695.

34. Perrot C. H, 1989. Le système de gestion de la pêche en lagune Aby au XIXe siècle (Côte d'Ivoire), Cahiers Sciences Humaines, vol. $25 \mathrm{n}^{\circ}$ 
1-2. La pêche: enjeux de développement et objet de recherche, IRD Bondy, Paris, p. 177-188.

35. Pouomogne V., Brummett R.E. and Gatchouko M., 2010. Impacts of aquaculture development projects in western Cameroon. Journal of applied aquaculture, 22, 93-108.

36. Pouomogne V. and Pemsl D.E., 2008. Recommendation domains for pond aquaculture. Country Case study: Development and status of freshwater aquaculture in Cameroon- Worldfish Center Studies and Reviews $\mathbf{N}^{\circ}$ 1871, The WorldFish Center (Ed.), Penang (Malaysia), $60 \mathrm{p}$.

37. Tomedi Eyango M.T., Mikolasek O. \& Pouomogne V., 2009. Diagnostic de l'insertion de la pisciculture dans les exploitations familiales agricoles du département de la Ménoua, Ouest Cameroun. Sci. Agron. \& Dév., 5(2), 13-22.

38. Wautelet J.M., 1995. Disparités régionales et différenciation des paysanneries au Cameroun. Tiers-Monde, 36(141), 87-101.

39. Zhang, R 1995. Scientific and technological development of rice-fish culture in China, p 23-30. In KT Mackay (ed). International Development Research Centre (IDRC), Ottawa, Canada, 276p 